\title{
CompuEyte
}

\section{Quantitative imaging cytometry: instrumentation of choice for automated cellular and tissue analysis}

Laser scanning cytometry (LSC) is the technology of choice for automated, quantitative high-content, high-throughput analysis of heterogeneous cellular samples and tissue specimens. LSC complements flow cytometry, allowing analysis of samples attached to solid substrates, such as adherent cells and tissue specimens. It also augments confocal imaging systems, making it far easier to perform population and subpopulation analysis and gather quantitative data on thousands of cells. LSC offers many advantages compared with camera-based imaging systems. Covering a broad range of applications, LSC technology is 'must have' instrumentation in every core flow cytometry and imaging facility and in all laboratories that study adherent cells and tissues.

\section{CompuCyte and its products}

CompuCyte Corporation (Westwood, Massachusetts) is a pioneer in quantitative imaging cytometry (QIC). CompuCyte's iGeneration laser scanning cytometry (LSC) instruments (iCyte ${ }^{\circledR}, \mathrm{iCys}^{\circledR}$ and $\mathrm{iColor}^{\circledR}$ ) provide accurate, precise and quantitative measurements of cellular and tissue constituents, coupling this quantification with fluorescence, absorbance and light-scatter imaging for exceptional analytic capabilities. LSC's versatility opens up a wide range of applications, enabling analysis of subnuclear, nuclear, cytoplasmic and intercellular signaling, as well as tissue and tissue microarrays (TMAs), in basic research, drug development, biomarker discovery and clinical trials.

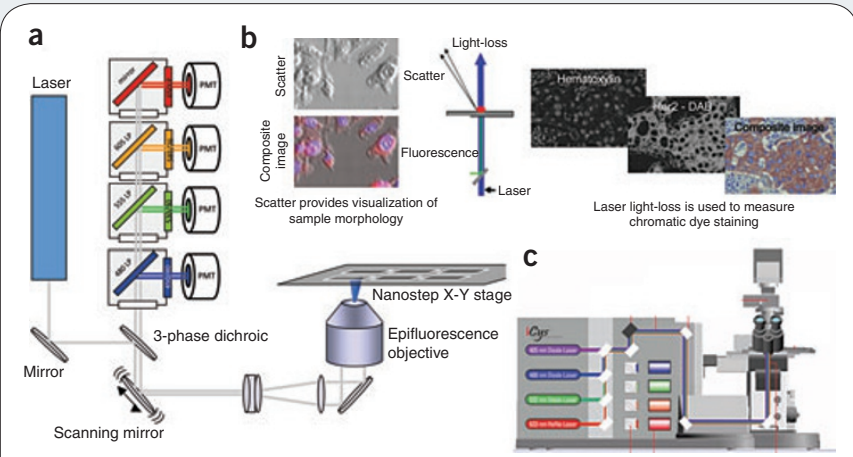

Figure 1 | Schematic diagrams of iGeneration technology. (a) Fluorescent measurement optical path. (b) Absorbance/scatter optical path and examples of images. (c) iCys system.

Melvin Henriksen

CompuCyte Corporation, Westwood, Massachusetts, USA

Correspondence should be addressed to M.H. (mhenriksen@compucyte.com).
Laser scanning cytometer configuration

An LSC cytometer is equipped with up to four lasers chosen from a palette of six possible wavelengths: $405 \mathrm{~nm}, 488 \mathrm{~nm}, 532 \mathrm{~nm}, 594$ $\mathrm{nm}, 561 \mathrm{~nm}$ and $633 \mathrm{~nm}$. Up to four photomultiplier tubes (PMTs), each detecting a specific wavelength range, collect fluorescence from excitation by scanning lasers. As the laser light intersects the sample, scattered or transmitted light is simultaneously directed to one or more solid-state photosensors. The photosensor and PMT signals are converted into 14-bit pixel values that are then assembled into highresolution images. The instruments' unique design allows simultaneous use of fluorescent and chromatic dyes (Fig. 1).

\section{Quantitative data analysis}

'Events' such as cells, nuclei or other subcellular structures are identified and segmented on the basis of their fluorescence, chromatic or scatter signal level (Fig. 2). From the segmented events, a variety of quantitative data are calculated: area, integrated signal, max pixel, peripheral integral, circularity, perimeter, $x$ and $y$ coordinates, and more. The numerical values are displayed in scattergrams and
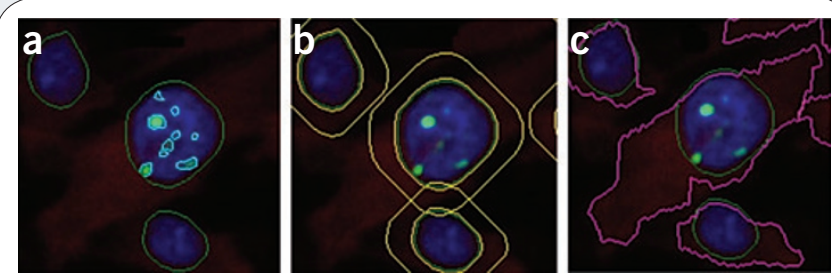

Figure 2 | Thresholding and segmentation routines. (a-c) Multiple

segmentation routines: nuclear and subnuclear (a); peripheral, used to sample a defined area around the nucleus (b); and cytoplasmic (c) 
histograms, allowing relationships among the various features, and between the features and the images, to be explored easily. For samples in which it is difficult to establish boundaries between individual cellular events—such as confluent adherent cells or tumorous tissuerandom sampling may be applied.

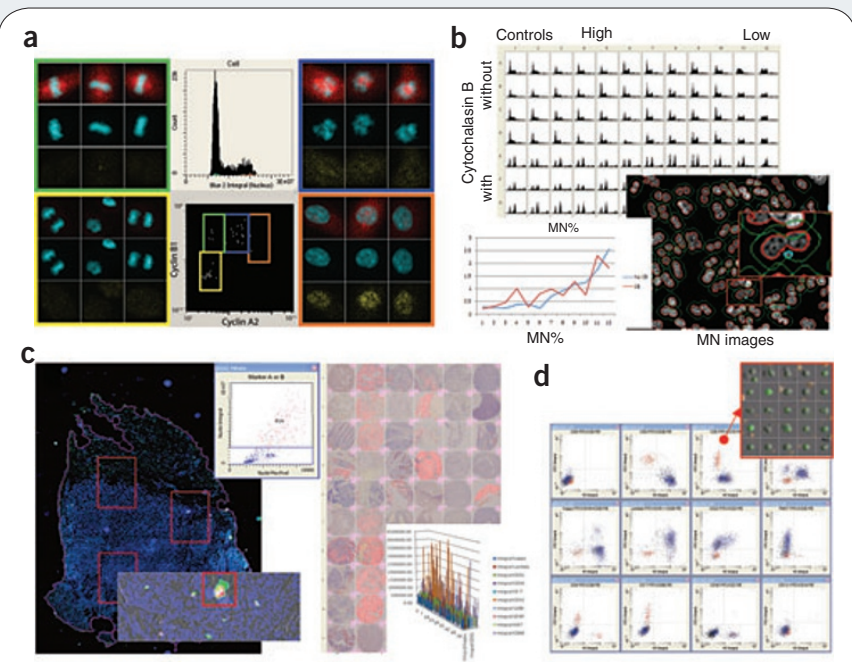

Figure $\mathbf{3}$ | Example applications of LSC technology. (a) Advanced cell cycle analysis: HuVEC cells stained with DAPI and antibodies for cyclin B1, cyclin $\mathrm{A} 2$ and $\mathrm{pH} 3$. Phases of mitosis are determined from levels of cyclin B2 and cyclin A1.Gating is confirmed by image galleries. (b) Micronucleus (MN) assay: quantitative and image data showing cell cycle effects of etoposide on $\mathrm{CHO}$ cells. (c) Automated fluorescently labeled tissue section (left) and TMA (right) analysis. An initial rapid overview scan identifies areas for high-resolution scanning. Quantitative data on marker expression is generated automatically from high-resolution scans. (d) Cell surface immunophenotyping. Scattergram panel shows cells single and double positive for cell surface markers. Inset, gallery of representative cells for one subpopulation.

\section{Variable image resolution and throughput scanning option} Image resolution is determined by both objective-lens magnification and stage resolution. Stage resolution can be selected (from 0.05 to $20 \mu \mathrm{m}$ ) to meet the needs of a particular assay, balancing spatial resolution, quantitative precision and throughput.

\section{iGeneration performance characteristics}

High precision: allows collection of quantitative signal through the full depth of the specimen, enabling total signal quantification. (DNA check bead coefficient of variations $(\mathrm{CVs})<2.5 \%$.)

High dynamic range: PMT detectors allow exposure to be set to match sample fluorescence across a wide range of intensities, without affecting scan time.

High analytic sensitivity: below 1,000 molecules of equivalent fluorochromes (MEFs). LSC's spot-scan design, illuminating only the sample area being measured, reduces background signal and allows better separation of low signal from background.

High-content analysis and multiplexing: PMTs and photodiodes collect signals simultaneously; image acquisition time is independent of the number of acquisition channels. An unlimited number of calculations can be derived from primary channels.

\section{Applications}

The technology's versatility allows many cellular and tissue-based applications throughout the biomedical sciences and pharmaceutical development ${ }^{1}$.

Cell cycle analysis: the cell cycle can be tracked by direct measurement of the total fluorescence of a nuclear dye, which is proportional to the DNA content. The DNA content histogram shows cell populations in sub-G1, G1, G2 and S phases. Note the sharp G1 peak in Figure 3a, a classic and robust measure of the quality of DNA content analysis. Cell cycle analysis can be multiplexed with additional markers of proliferation or cell cycle phase-for example, phospho-histone $\mathrm{H} 3$, cyclin $\mathrm{B} 1$ and cyclin $\mathrm{A} 2$ - enabling a much finer segregation of mitotic cells (Fig. 3a).

Such precise DNA quantification is impossible or impractical with confocal microscopy and many camera-based systems. LSC's capabilities, along with its easy-to-use and comprehensive methods of relating graphic, image and numerical data, make it a preferred choice for studies requiring simple or multiplexed cell cycle analysis of adherent cells.

DNA damage studies: these are often related to and combined with cell cycle analysis. Examples include measurement of $\mathrm{H} 2 \mathrm{AX}$ phosphorylation as an indicator of DNA double-strand breaks, pATM, 53BP1 and quantification of changes in nuclear morphology (micronucleus formation (Fig. 3b), nuclear buds and nuclear bridges).

Automated tissue and tissue microarray analysis: from routine two- to three-color chromatically stained specimens to cutting-edge multicolor colocalization and in situ protein expression analysis, iGeneration technology uniquely enables simultaneous evaluation of fluorescently and chromatically stained tissue sections, TMAs and core biopsies (Fig. 3c) ${ }^{2}$.

Cell surface and tissue immunophenotyping: when only a small number of cells are available-often the case with fine-needle aspirates or tissue samples-immunophenotyping cannot be done with traditional flow cytometry. LSC is an ideal methodology in these instances. More than 20 antigens can be assessed in the same analysis using pairs of FITC- and PE-conjugated antibodies on a 12-lane proprietary slide (Fig. 3d). While correlating well to flow cytometry ${ }^{3}$, LSC offers significant advantages: cell immunophenotype can be linked to cell morphology, and cells can be reprocessed in situ with cytoplasmic, immunological or genetic markers.

1. Harnett, M. Laser scanning cytometry: understanding the immune system in situ. Nat. Rev. Immunol. 7, 897-904 (2007).

2. Peterson, R.A. et al. Applications of laser scanning cytometry in immunohistochemistry and routine histopathology. Toxicol. Pathol. 36, 117132 (2008)

3. Al-Za'abi, A.M. et al. Equivalence of laser scanning cytometric and flow cytometric immunophenotyping of lymphoid lesions in cytologic samples. Am. J. Clin. Pathol. 129, 780-785 (2008).

This article was submitted to Nature Methods by a commercial organization and has not been peer reviewed. Nature Methods takes no responsibility for the accuracy or otherwise of the information provided. 